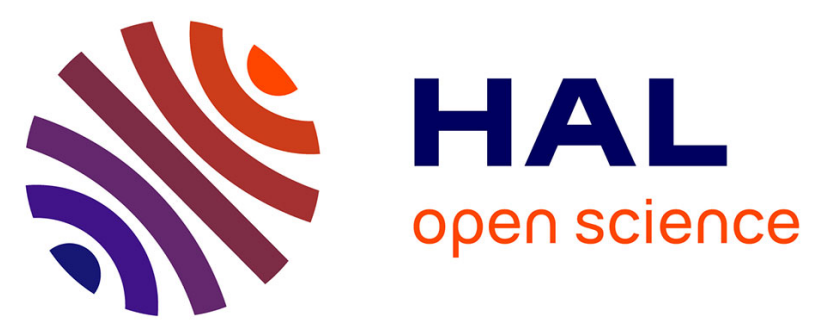

\title{
Effect of functionalization on the electrostatic charging, tunneling, and Raman spectroscopy of epitaxial graphene
}

Jeongmin Hong, Sandip Niyogi, Elena Bekyarova, Mikhail E. Itkis, Palanisamy Ramesh, Claire Berger, Walt A. de Heer, Robert C. Haddon, Sakhrat Khizroev

\section{To cite this version:}

Jeongmin Hong, Sandip Niyogi, Elena Bekyarova, Mikhail E. Itkis, Palanisamy Ramesh, et al.. Effect of functionalization on the electrostatic charging, tunneling, and Raman spectroscopy of epitaxial graphene. Journal of Vacuum Science \& Technology B Microelectronics and Nanometer Structures, 2012, 30, pp.03D103. 10.1116/1.3693417 . hal-01002905

\section{HAL Id: hal-01002905 https://hal.science/hal-01002905}

Submitted on 7 Jun 2014

HAL is a multi-disciplinary open access archive for the deposit and dissemination of scientific research documents, whether they are published or not. The documents may come from teaching and research institutions in France or abroad, or from public or private research centers.
L'archive ouverte pluridisciplinaire HAL, est destinée au dépôt et à la diffusion de documents scientifiques de niveau recherche, publiés ou non, émanant des établissements d'enseignement et de recherche français ou étrangers, des laboratoires publics ou privés. 


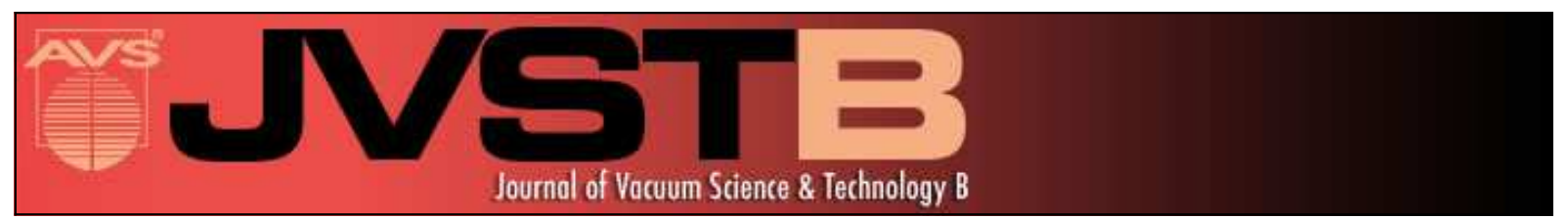

\section{Effect of functionalization on the electrostatic charging, tunneling, and Raman spectroscopy of epitaxial graphene}

Jeongmin Hong, Sandip Niyogi, Elena Bekyarova, Mikhail E. Itkis, Palanisamy Ramesh, Claire Berger, Walt A. deHeer, Robert C. Haddon, and Sakhrat Khizroev

Citation: Journal of Vacuum Science \& Technology B 30, 03D103 (2012); doi: 10.1116/1.3693417

View online: http://dx.doi.org/10.1116/1.3693417

View Table of Contents: http://scitation.aip.org/content/avs/journal/jvstb/30/3?ver=pdfcov

Published by the AVS: Science \& Technology of Materials, Interfaces, and Processing

\section{Articles you may be interested in}

Effect of the polarity of carbon-fluorine bonds on the work function of plasma-fluorinated epitaxial graphene Appl. Phys. Lett. 101, 111602 (2012); 10.1063/1.4752443

Evidences of electrochemical graphene functionalization and substrate dependence by Raman and scanning tunneling spectroscopies

J. Appl. Phys. 111, 114306 (2012); 10.1063/1.4725489

Pinned and unpinned epitaxial graphene layers on SiC studied by Raman spectroscopy

J. Appl. Phys. 111, 114307 (2012); 10.1063/1.4721673

X-ray radiation effects in multilayer epitaxial graphene

Appl. Phys. Lett. 99, 232102 (2011); 10.1063/1.3665953

Substrate doping effects on Raman spectrum of epitaxial graphene on SiC

J. Appl. Phys. 107, 034305 (2010); 10.1063/1.3283922

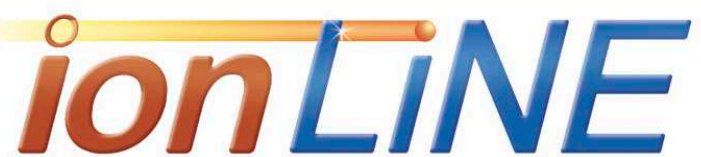

Select Si Ge Au and more for Advanced Nanofabrication www.raith.com

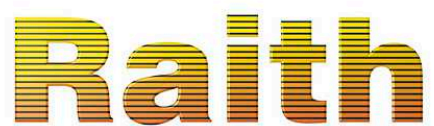




\title{
Effect of functionalization on the electrostatic charging, tunneling, and Raman spectroscopy of epitaxial graphene
}

\author{
Jeongmin Hong a) \\ Florida International University, Department of Electrical and Computer Engineering, 10555 West Flagler \\ Street, Miami, Florida 33174 and Univeristy of California-Riverside, Department of Electrical Engineering, \\ 900 University Avenue, Riverside, California 92521
}

Sandip Niyogi, Elena Bekyarova, Mikhail E. Itkis, and Palanisamy Ramesh

University of California-Riverside, Department of Chemistry, Department of Chemical \& Environmental

Engineering and Center for Nanoscale Science and Engineering, 900 University Avenue, Riverside, California 92521

Claire Berger

Institut Neel, CNRS/UJF 25 rue des Martyrs, BP166, 38042 Grenoble Cedex 9, France and Georgia Institute of Technology, School of Physics, 837 State Street Northwest, Atlanta, Georgia 30332

Walt A. deHeer

Georgia Institute of Technology, School of Physics, 837 State Street Northwest, Atlanta, Georgia 30332

Robert C. Haddon

University of California-Riverside, Department of Chemistry, Department of Chemical \& Environmental

Engineering and Center for Nanoscale Science and Engineering, 900 University Avenue, Riverside,

California 92521

Sakhrat Khizroev

Florida International University, Department of Electrical and Computer Engineering, 10555 West Flagler Street, Miami, Florida 33174 and University of California-Riverside, Department of Electrical Engineering, 900 University Avenue, Riverside, California 92521

(Received 11 December 2011; accepted 16 February 2012; published 12 March 2012)

The authors report the effects of radical functionalization on the electrostatic force microscopy (EFM), the scanning tunneling spectra (STS), and Raman spectroscopy of epitaxial graphene. The EFM studies show the existence of layer-dependent trapped charges in the pristine graphene. The uniform enhancement of energy gap is observed through STS. Raman spectra show nonuniformly distributed D-band intensities throughout the functionalized sample as a result of the inhomogeneous distribution of covalent bonds to the graphene sheets. The functionalization chemistry has a marked effect on the homogeneity of the electrostatic charge and leads to an increase of the energy of the band gap. (C) 2012 American Vacuum Society. [http://dx.doi.org/10.1116/1.3693417]

\section{INTRODUCTION}

Due to the combination of unique properties such as the ballistic transport at room temperature, the presence of quantum Hall effect and minimum conductivity, graphene has brought significant attention in the scientific community. ${ }^{1-4}$ Epitaxial graphene (EG) is especially attractive to the electronic industry because of the potential for wafer scale production. Moreover, recent experimental results have demonstrated that covalent modification can effectively modify the electronic and magnetic properties of graphene..$^{5-12}$ Thus, the chemical route utilizing covalent carbon-carbon $(\mathrm{C}-\mathrm{C})$ bond formation involving the basal plane carbon atoms provides an alternative approach to control the physical properties of epitaxial graphene grown on $\mathrm{SiC}$ wafers of macroscopic dimensions without physical patterning. Because of the inherent advantages of $\mathrm{C}-\mathrm{C}$ covalent bonds in materials applications, there has been an extensive effort to understand the chemical conditions that lead to stable states for engineer-

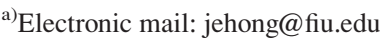

ing the energy gap, inducing ferromagnetism, and patterning electronic circuits of $\mathrm{sp}^{2}$-bonded carbon materials.

The EG on silicon carbide substrates offers the advantage that high quality layers can be grown on large area substrates. However, it is difficult to precisely define the local thickness of the graphene due to the complex morphology of the $\mathrm{SiC}$ substrate and inhomogeneity of the graphene growth. The controlled growth of EG on $\mathrm{SiC}$ crystals has allowed for the application of covalent chemistry to the modification of the electronic structure of graphene in its chemically pure, crystalline state. ${ }^{1-4}$ Understanding the chemical reactivity of epitaxial graphene is important for the controlled modification of its semiconducting and magnetic properties. The optimization of the covalent chemistry of graphene requires development of analytical techniques to quantify $\mathrm{C}-\mathrm{C}$ bond formation. ${ }^{8,11}$ Further systematic methods are required for studying the changes in the electric and magnetic properties of graphene as a function of its characteristics and chemical reactions. ${ }^{12-18}$ The electrostatic force microscopy (EFM) technique was previously used for studies of graphite, exfoliated graphene (XG) on $\mathrm{SiO}_{2}$, and the EG. ${ }^{19-21}$ 
In this study, we use EFM to investigate the local nature of the samples as a result of electrostatic charging, particularly through the measurements of the surface potentials before and after nitrophenyl functionalization of epitaxial graphene. Simultaneous image measurements allow us to match the observed local surface potentials with the EG surface topology. The tunneling spectroscopy provides a complementary study of local I-V characteristics of the samples. Raman spectral area map gives local properties, particularly related to distributions of frequency, lineshape, and linewidth.

\section{EXPERIMENT}

We used graphene samples with average thickness of five layers, as measured with an ellipsometer. The EG samples were grown on the $\mathrm{C}$-face of $4 \mathrm{H}-\mathrm{SiC}$ wafers (Cree, Inc.), which were prepared by solid-state decomposition in vacuum. ${ }^{1,22}$ The method involves an inductively heated vacuum furnace in which $3.5 \mathrm{~mm} \times 4.5 \mathrm{~mm} \times 0.3 \mathrm{~mm}$ sized SiC substrates, are heated to about $1400^{\circ} \mathrm{C}$. In this process, Si sublimes to produce carbon-rich surfaces that subsequently graphitize. The graphitization produces epitaxially ordered stacked layers of graphene, with a high structural coherence length. ${ }^{18}$

The epitaxial graphene (EG) substrate was immersed in a solution of $10 \mathrm{mM}$ 4-nitrophenyl diazonium (NPD) tetrafluoroborate and $0.1 \mathrm{M}$ tetrabutylammonium hexafluorophosphate ([Bu4N]PF6]) in degassed acetonitrile (ACN) for $20 \mathrm{~h}$ in a glove box in the absence of light, after which it was removed and washed with ACN and acetone. ${ }^{8}$ This method provides a gentle route for the covalent attachment of nitrophenyl groups to the basal plane carbon atoms in the accessible top surface of graphene.

The EFM was performed in a noncontact mode with a Veeco Nanoscope IIIa system. The measurements were conducted in a dynamic lift mode with a lift-off distance of 30 $\mathrm{nm}$. The cantilever is mechanically driven at its resonance frequency, and the phase shift of the cantilever oscillation is measured as a function of the tip position. The capacitive coupling of the tip and sample results in a phase shift.

The I-V tunneling spectra were acquired using a Veeco Nanoscope IV system under ambient conditions after verifying that tips were adequately engaged throughout the entire STM image scan. The measured tunneling I-V characteristics correspond to the regions of multilayered graphene. The tunneling current affects the I-V characteristics under study. Increasing the current set point ensures the shortest separation between the tip and the sample to minimize the tunneling current contribution.

Raman spectra were collected in a Nicolet Almega XR Raman microscope using $532 \mathrm{~nm}$ laser excitation, with a 633 lines $/ \mathrm{mm}$ and a 2400 lines $/ \mathrm{mm}$ grating for the low (6-10 $\left.\mathrm{cm}^{-1}\right)$ and high $\left(1.5-2.5 \mathrm{~cm}^{-1}\right)$ resolution spectra, respectively. ${ }^{11}$ The reflective face of a gold plated glass microscope slide was used as a mirror below the optically transparent multilayered graphene-SiC substrates. The maximum incident laser power on the sample was $0.4 \mathrm{~mW}$. A
$50 \times$ or a $100 \times$ objective was used with the microscope to define the laser spot size $(0.7 \mathrm{~mm})$ on the sample. A motorized $x-y-z$ stage allowed the collection of Raman spectra as a function of the focal plane along the c-axis of the samples. This was particularly useful for collecting Raman spectra of the multilayered graphene-SiC samples while minimizing the signal from the $\mathrm{SiC}$ substrate. To obtain a spectrum without the $\mathrm{SiC}$ signals, the spectrum of $\mathrm{SiC}$ was collected at every sample spectral position by focusing on the lower substrate using identical spectral parameters. The $\mathrm{SiC}$ spectrum was then subtracted from the spectrum of the sample.

\section{RESULTS AND DISCUSSION}

To investigate the effects of the nitrophenyl functionality on the electric charge of the graphene sheets, we acquired images from the same region before and after chemical functionalization. Charge transfer from the $\mathrm{SiC}$ substrate to the graphene results in a rigid shift of the electronic levels and the work function. ${ }^{23}$ The EFM phase images of the buffer layer, Oth layer (BL) are distinct due to the adsorbates, while the single layer graphene (1LG) regions are also clearly distinguishable. ${ }^{21}$ The frequency of the Raman D-band is characteristic and arises from the localized, structural defects in the $\mathrm{sp}^{2}$ conjugated carbon network due to the chemical functionalization of the basal plane of graphene. The reaction occurs by spontaneous electron transfer from graphene to p-nitrobenzenediazonium tetrafluoroborate and ultimately results in the formation of a $\mathrm{C}-\mathrm{C}$ bond between graphene and the nitrophenyl group and the rehybridization of the graphene carbon atoms from $\mathrm{sp}^{2}$ to $\mathrm{sp}^{3}$

From the measured sample surface potential, we observed patches with positive and negative uniform trapped charges at positive and negative bias voltages, respectively, in a $\pm 2 \mathrm{~V}$ range, as shown in Fig. 1(A). A previous study ${ }^{11}$ with Raman spectroscopy explained this as the charge trapped in the buffer layer (BL) directly onto the $\mathrm{SiC}$ substrate and the single-layer graphene (1LG), as shown in Fig. 1(A) in red and blue arrows, respectively.

Because of the large difference of thermal expansion coefficients of $\mathrm{SiC}$ and $\mathrm{EG},{ }^{24}$ the cooling of the sample from the growth temperature induces compressive strain, which may result in the adsorbates at the $\mathrm{BL}^{25}$ As shown in Fig. 1(A), 1LG and BL show a typical behavior with trapped charges as a function of the bias voltage. The electrostatic phase is zero when the tip bias and electrostatic surface potentials are equal. The reversible discrepancy shifted to the right shows the values of the electrostatic surface potential, which varies with the number of graphene layers. ${ }^{21} \mathrm{~A}$ change in the electrostatic charge effects in the different regions due to work function varies as a function of graphene thickness.

The measured phase shift was calculated from the relationship between the phase shift of the driving force and the cantilever oscillation and tip and sample capacitive coupling, $\Phi(x, y)=-(Q / 2 k) C^{\prime \prime}(h)\left(V_{\text {tip }}-V_{s}(x, y)\right)^{2}$ where $C^{\prime \prime}(h)$ is the second derivative of the difference between the tip and sample capacitance and the tip and a bare substrate capacitance 


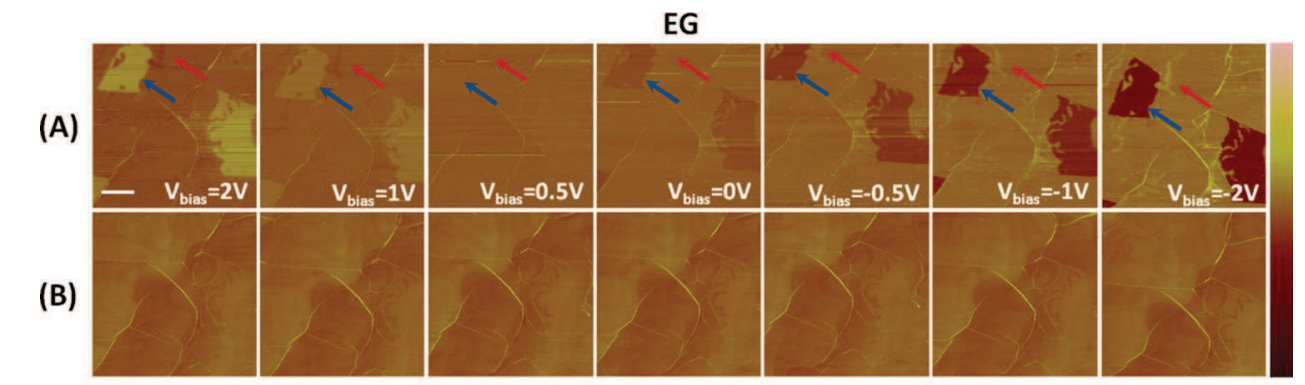

(C)

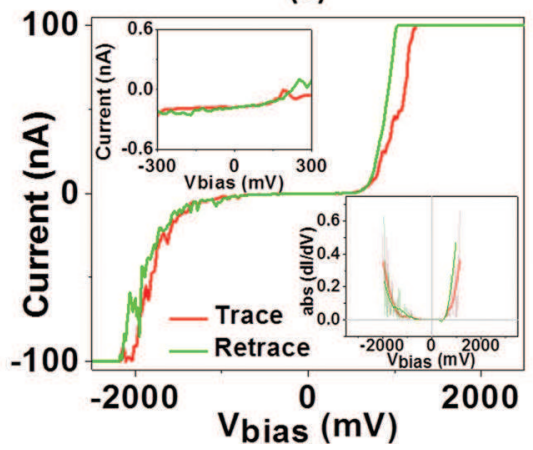

(D)

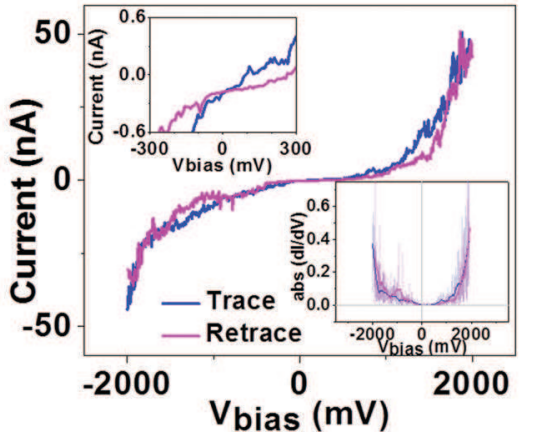

Fig. 1. (Color) EFM images: (A) EFM phase and (B) topography of pristine graphene. The scale bars in phase and topography are $2 \mu \mathrm{m}$. The color bar of (A) and (B) is $5^{\circ}$ and $40 \mathrm{~nm}$, respectively. STS measurements: The $\mathrm{I}(\mathrm{V})$ curves of two different random regions (C) and (D) of pristine graphene. Insets on the left show corresponding absolute conductance $(\mathrm{dI} / \mathrm{dV})$ curves of the region; set point voltage $\sim 80 \mathrm{mV}$; set point current $\sim 0.2 \mathrm{nA}$.

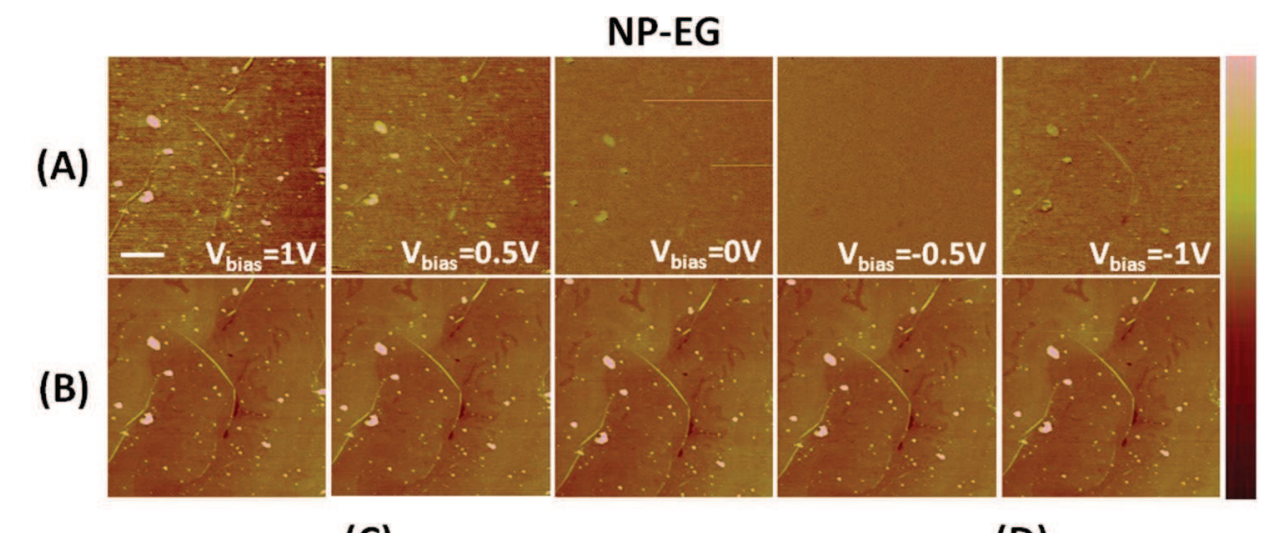

(C)

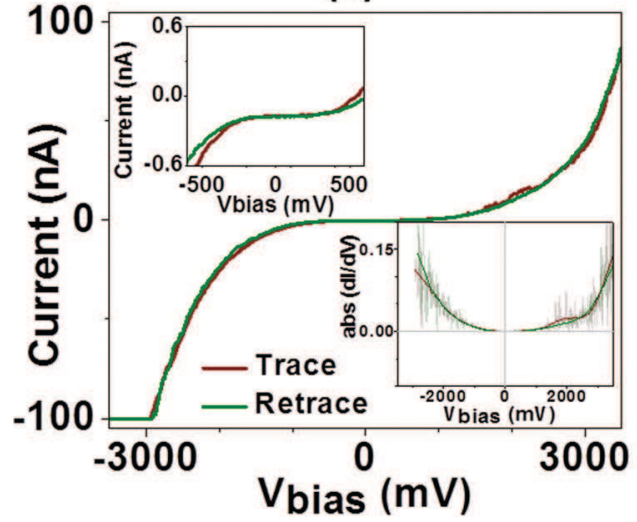

(D)

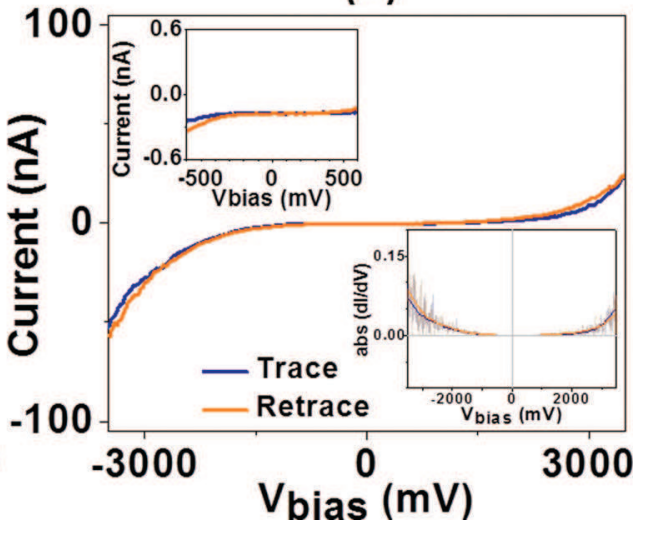

Fig. 2. (Color) EFM images: (A) EFM phase of NP-EG and (B) NP-EG topography, respectively. The scale bars are $2 \mu \mathrm{m}$. The color bar of (A) and (B) is $1^{\circ}$ and $40 \mathrm{~nm}$, respectively. STS measurements: The I(V) curves of a region (C) and the other region (D) of NP-EG. Insets on the left indicate absolute conductance $(\mathrm{dI} / \mathrm{dV})$ curves of two regions; set point voltage $\sim 80 \mathrm{mV}$; set point current $\sim 0.2 \mathrm{nA}$. 
as a function of $h$, and $V_{s}(x, y)$ is the local electrostatic potential on the sample surface. Likewise, two factors could contribute to an EFM signal: (1) the capacitance between the tip and sample that is determined by the tip and sample distance, the materials used, and the shapes of the tip and sample and (2) the potential difference between the tip and sample. ${ }^{20}$ The potential difference depends linearly on the sample potential when a constant voltage is applied to the tip. If the signal is due to a capacitance variation, the EFM images obtained using a positive or negative tip bias voltage should be the same. When the potential difference equals the bias voltage applied through the tip, the phase might be zero because the electrostatic potential might be zero. The nonzero electrostatic charge effects are from different work functions with graphene thickness and those of tip/sample.

As shown in Fig. 2(A), covalent chemical functionalization led to the disappearance of the patches due to the inhomogeneous distribution of trapped charge over the entire EG sample. The nonuniform charge distribution also correlates with the differences in the chemical reactivity, as indicated by the variation of the intensity of the Raman D-band. ${ }^{11}$ The uniformity of the electrostatic charge also points to a uniform work function on the graphene surface after chemical functionalization. The contrast of the EFM signals is indicative of the capacitance variation and consequently, the uniform EFM

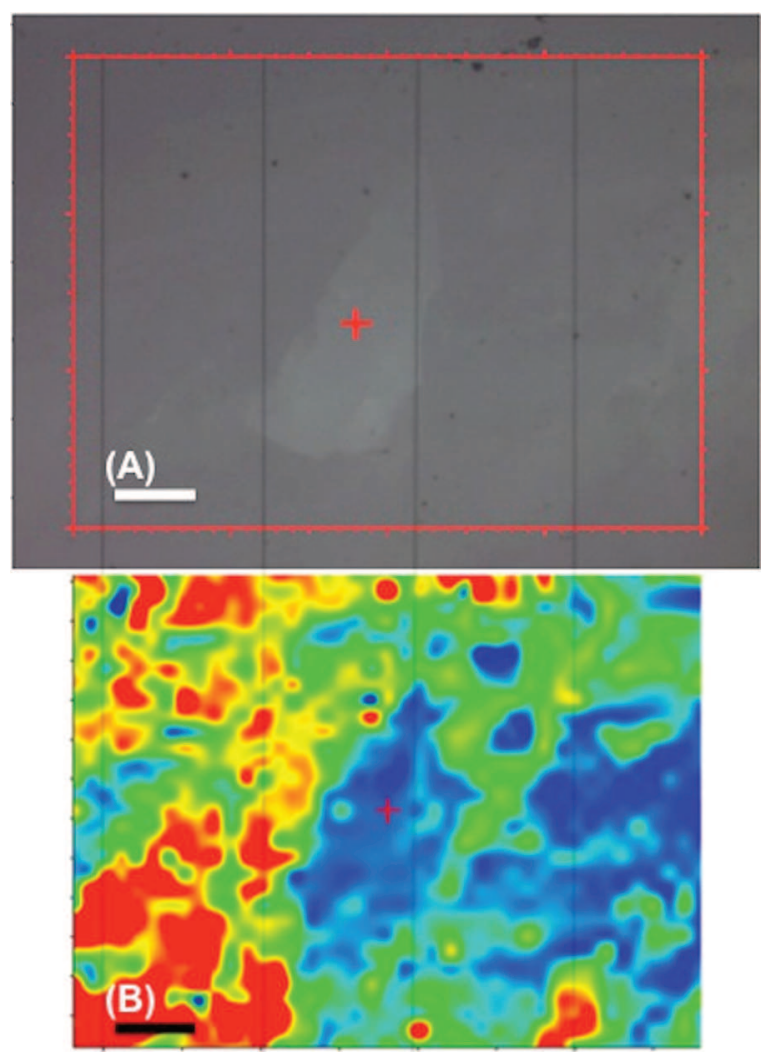

Fig. 3. (Color) Raman map: (A) Optical microscopy image of the NPfunctionalized epitaxial graphene on $\mathrm{SiC}(\mathrm{C}$-face $)$ sample described in Figs. 1 and 2, showing dark and light domains. (B) Spectral map of the Raman D-band $\left(1340 \mathrm{~cm}^{-1}\right)$, over the same region (scan size $\sim 80 \mu \mathrm{m}$ $\times 60 \mu \mathrm{m}$ ), using $532 \mathrm{~nm}$ excitation. The red color corresponds to higher intensity while the blue is lowest intensity of the Raman D-band. The black and white scale bars are $10 \mu \mathrm{m}$. signal in the $\pm 1 \mathrm{~V}$ bias voltage range has a characteristic parabolic dependence after the functionalization. This indicates that single-layer graphene should be more reactive toward electron-transfer reactions as the graphene work function increases with the number of layers, although additional factors come into play such as the nature of the interface states at the $\mathrm{SiC}$ and presence of trapped charge.

Atomically resolved STM imaging of the EG under ambient conditions provides us with the information about the correlation of the functional group density and the periodicity in microscopic samples where the transport characteristics can be measured. ${ }^{26}$ We conducted scanning tunneling spectra (STS) measurements under ambient conditions to observe the local nature of its I-V characteristics. Figures 1(C), 1(D), 2(C), and 2(D) show the measured I-V curves in two arbitrary local regions in the pristine and functionalized samples, respectively. The lower energy gap opening in the pristine sample (compared to the functionalized sample) may be explained with either the presence of defects or by the $\mathrm{SiC}$-substrate-induced gap opening. ${ }^{27-29}$

Previously, various morphological inhomogeneties have been observed in the epitaxial graphene samples that give rise to variations in the physical properties at a microscopic scale. ${ }^{12,30,31}$ Figure 3 shows that surface inhomogeneties observed in optical microscopy as a variation in the reflected light intensity [Fig. 3(A)] relate to a difference in the chemical reactivity that can be estimated from the intensity and spatial density of the Raman D-band (red color). The nitrophenyl functionalization reaction is activated by spontaneous electron transfer from graphene and it is known from electron scattering measurements that the electron affinity of graphene increases with the number of layers, which would suggest a decreased reactivity towards the nitrophenyl reaction and increased surface inhomogeneity of functionalized sites. ${ }^{11,32,33}$

\section{SUMMARY AND CONCLUSIONS}

In summary, we draw the following conclusions. We find that the nonuniform Raman spectra of pristine EG correlate with the number of layers dependent effects. Also, the nonuniformity of Raman D-peaks after chemical functionalization results from the variations in the reactivity of EG toward nitrophenyl. The EFM results suggest that the chemical functionalization reaction makes the electrostatic charging effects uniform over the entire graphene surface. The trapped charges (which are a function of the graphene thickness) may affect the density of the covalent attachment of the chemical functionality resulting from the spontaneous electron transfer. In addition, the STS measurements indicate that the radical functionalization induces an increase in the energy gap at least $0.4 \mathrm{eV}$.

\section{ACKNOWLEDGMENTS}

This material is based on research cosponsored by the National Science Foundation (NSF) under Contracts Nos. 005084-002 and 0824019 and DARPA/Defense Microelectronics Activity (DMEA) under agreement No. H94003-092-0904. 
${ }^{1}$ C. Berger et al., J. Phys. Chem. B 108, 19912 (2004).

${ }^{2}$ K. S. Novoselov, A. K. Geim, S. V. Morozov, D. Jiang, Y. Zhang, S. V. Dubonos, I. V. Grigorieva, and A. A. Firsov, Science 306, 666 (2004).

${ }^{3}$ Y. W. Tan, Y. Zhang, H. L. Stormer, and P. Kim, Eur. Phys. J. Spec. Top. 148, 15 (2007).

${ }^{4}$ K. I. Bolotin, K. J. Sikes, J. Hone, H. L. Stormer, and P. Kim, Phys. Rev. Lett. 101, 096802 (2008).

${ }^{5}$ M. Y. Han, B. Oezyilmaz, Y. Zhang, and P. Kim, Phys. Rev. Lett. 98, 206805 (2007).

${ }^{6}$ I. Meric, M. Y. Han, A. F. Young, B. Oezyilmaz, P. Kim, and K. L. Shepard, Nat. Nano. 3, 654 (2008).

${ }^{7}$ Y. H. Lu and Y. P. Feng, J. Phys. Chem. C 113, 20841 (2009).

${ }^{8}$ E. Bekyarova, M. E. Itkis, P. Ramesh, C. Berger, M. Sprinkle, W. A. de Heer, and R. C. Haddon, J. Am. Chem. Soc. 131, 1336 (2009).

${ }^{9}$ E. Bekyarova, M. E. Itkis, P. Ramesh, and R. C. Haddon, Phys. Status Solidi (RRL) 3, 184 (2009).

${ }^{10}$ P. Ramesh, M. E. Itkis, E. Bekyarova, F. Wang, S. Niyogi, X. Chi, C. Berger, W. A. de Heer, and R. C. Haddon, J. Am. Chem. Soc. 132, 14429 (2010).

${ }^{11}$ S. Niyogi et al., Nano Lett. 10, 4061 (2010).

${ }^{12}$ J. Hong et al., Small 7, 1175 (2011).

${ }^{13}$ S. Ryu, M. Y. Han, J. Maultzsch, T. F. Heinz, P. Kim, M. Steigerwald, and L. E. Brus, Nano Lett. 8, 4597 (2008).

${ }^{14}$ H. Liu, S. Ryu, Z. Chen, L. Michael, M. L. Steigerwald, C. Nuckolls, and L. E. Brus, J. Am. Chem. Soc. 131, 17099 (2009).

${ }^{15}$ J. Jung and A. H. MacDonald, Phys. Rev. B 80, 235417 (2009).

${ }^{16}$ J. A. Robinson, C. P. Puls, N. E. Staley, J. P. Stitt, M. A. Fanton, K. A. Emtsev, T. Seyller, and Y. Liu, Nano Lett. 9, 964 (2009).
${ }^{17}$ T. Lohmann, K. von Klitzing, and J. H. Smet, Nano Lett. 9, 1973 (2009). ${ }^{18}$ W. A. de Heer et al., J. Phys. D 43, 374007 (2010).

${ }^{19}$ Y. Lu, M. Munoz, C. S. Steplecaru, C. Hao, M. Bai, N. Garcia, K. Schindler, D. Spoddig, and P. Esquinazi, Phys. Rev. Lett. 98, 269702 (2007).

${ }^{20}$ S. S. Datta, D. R. Strachan, E. J. Mele, and A. T. C. Johnson, Nano Lett. 9, 7 (2009).

${ }^{21}$ T. Burnett, R. Yakimova, and O. Kazakova, Nano Lett. 11, 2324 (2011).

${ }^{22}$ W. A. de Heer et al., Solid State Commun. 143, 92 (2007).

${ }^{23}$ C. Mathieu, N. Barrett, J. Rault, Y. Y. Mi, B. Zhang, W. A. de Heer, C. Berger, E. H. Conrad, and O. Renault, Phys. Rev. B 83, 235436 (2011).

${ }^{24}$ N. Ferralis, R. Maboudian, and C. Carraro, Phys. Rev. Lett. 101, 156801 (2008).

${ }^{25}$ J. Rohrl, M. Hundhausen, K. V. Emtsev, T. Seyller, R. Graupner, and L. Ley, Appl. Phys. Lett. 92, 201918 (2008).

${ }^{26}$ S. Niyogi, E. Bekyarova, J. Hong, C. Berger, W. A. de Heer, S. Khizroev, and R. C. Haddon, J. Phys. Chem. Lett. 2, 2487 (2011).

${ }^{27}$ Y. Zhang, V. W. Brar, C. Girit, A. Zettl, and M. F. Crommie, Nat. Phys. 5, 722 (2009).

${ }^{28}$ M. Sprinkle et al., Phys. Rev. Lett. 103, 226803 (2009).

${ }^{29}$ C. Coletti, C. Riedl, D. S. Lee, B. Krauss, L. Patthey, K. von Klitzing, J. H. Smet, and U. Starke, Phys. Rev. B 81, 235401 (2010).

${ }^{30}$ D. K. Gaskill et al., ECS Trans. 19, 117 (2009).

${ }^{31}$ M. Sprinkle et al., J. Phys. D 43, 374006 (2010).

${ }^{32}$ F. M. Koehler, A. Jacobsen, K. Ensslin, C. Stampfer, and W. J. Stark, Small 6, 1125 (2010).

${ }^{33}$ A. Sinitskii, A. Dimiev, D. A. Corley, A. A. Fursina, D. V. Kosynkin, and J. M. Tour, ACS Nano 4, 1949 (2010). 